\title{
A Novel Design for Assembly Approach for Modified Topology of Industrial Products
}

\author{
G. Bala Murali*, B. B. V. L. Deepak and B. B. Biswal \\ Department of Industrial Design, National Institute of technology, Rourkela, India-769008
}

\begin{abstract}
Recent advancements in materials and manufacturing processes are becoming difficult in day-to-day times to meet the industrial needs. Assembly is one of the processes in manufacturing, which takeover $20 \%$ of overall cost in manufacturing [5]. The assembly of the parts still becomes difficult, if the product consists of parts with more intricate shape. Design For Assembly (DFA) has driven product designers towards minimizing the number of parts in a product to reduce the assembly efforts and manufacturing cost. Until now, there is no generalized method to obtain modified topology of the product by DFA concept. Many industries like Toyota, Sony and many more follow their own designed DFA methodology. Generalization of DFA concept to obtain the modified topology of the product involves high skilled user intervention and demands in deep knowledge of DFA principles. In this paper, an attempt is made to generate modified topology by generalizing the DFA concept. To generalize the DFA concept and to obtain the modified topology, the research work is mainly concentrated on four principles. 1) Material properties of the parts, 2) Relative motion between the parts, 3) Contact between the parts, 4) Functionality of the parts. Depending on these principles, a general methodology has been developed to obtain the modified topology of industrial products. The methodology has been successfully implemented on an industrial product to obtain modified topology with reduced part numbers.
\end{abstract}

Keywords: design for assembly; liaison data; material data; functionality data; relative motion data

(Submitted on May 20, 2017; Revised on October 10, 2017; Accepted on October 21, 2017)

(C) 2017 Totem Publisher, Inc. All rights reserved.

\section{Introduction}

Assembly is one of the major cost contribution operations in manufacturing. Many researchers are concentrating on how to achieve optimal assembly sequence while considering assembly predicates in order to reduce the manufacturing cost. Initially, knowledge based methods are implemented to achieve optimal assembly sequence [8,12,18]. As they need more search space, the implementation of Artificial Intelligence (AI) techniques drive researchers to implement soft computing techniques to achieve optimal assembly sequence $[9,17]$.

The implementation of AI techniques for assembly sequence planning lead to local optima solution [2,3,15]. Moreover, the AI techniques fail to consider all the assembly predicates for obtaining assembly sequences [11,16]. Hybrid techniques have been introduced to overcome the local optima problem by increasing the solution accuracy $[1,6,10]$. As hybrid techniques involve more complexity, the execution time for getting the optimal solution increases $[7,14]$.

Design for Assembly motivated researchers to reduce the assembly cost and time while decreasing the number of parts in the assembly. Initially, DFA concept was introduced by De Fazio et.al. in 1977, which is used to estimate the time and cost of the manual assembly compared to the automatic assembly. Later on they recognized that, the only way to reduce the manufacturing and assembly cost is by reducing the number of parts in the assembly. They developed a methodology that questions the customer regarding the material used, functionality of the product, maintenance required to the product and so on. Depending on the answers given by the customer, the modified product will be developed [4]. This method has a 
disadvantage that requires the customer should have the complete knowledge on the product and its function. So, a person with sound knowledge on the product can only handle this methodology. This method is very time consuming and requires more search space especially for more part assembly to store the data given by the customer. Until now, there is no generalized method for applying DFA concept [13]. The industries like Sony, Toyota, Benz and many more are adopting their own developed DFA methodologies to obtain the modified topology of the products.

In this paper, an attempt is made to generalize the DFA concept to achieve the modified topology of the product by reducing the number of levels of the assembly. The method is successfully implemented on the Boothroyd motor assembly to obtain the modified topology.

\section{DFA Predicates for Obtaining the Modified Topology}

To generate the modified topology of a given assembly, DFA predicates have been considered. These predicates are considered from the DFA principles to obtain the modified topology. In the present research work, four main DFA predicates have been chosen for merging the parts to obtain the reduced levels of the assembly.

To reduce the number of levels in the assembly, the part count is to be reduced by merging the appropriate parts. Merging of parts only takes place if the parts are made of same material; the parts should not move relative to each other and there should not be any functional disturbance between the parts. Therefore, these four criteria are considered as DFA predicates in this research work.

- Liaison data

- Material data

- Relative motion data

- Functionality data

DFA Predicates

\subsection{Liaison data.}

Liaison data provides the information about the contact between the parts. The data is written in the form of matrix size equal to part number in the assembly and which part is in contact with another. This data is automatically generated using Computer Aided Design (CAD) interface with CATIA V5 R17.

\subsection{Material data.}

Material data provides the information of parts having similar material. This data is written in the form of matrix size equal to part number in the assembly. This matrix helps in finding the similar material parts for merging. This data is automatically generated using CAD interface with CATIA V5 R17.

\subsection{Relative motion data.}

Relative motion data provides the information about the relative motion between parts. The data is written in the form of matrix size equal to part number in the assembly. This helps in finding the motion between the parts used during merging of the parts. This data is automatically generated using CAD interface with CATIA V5 R17.

\subsection{Functionality data.}

Functionality data provides the information about the operational disturbance of the merging parts. The data is written in the form of matrix size equal to part number in the assembly. This data is automatically generated using CAD interface with CATIA V5 R17.

\section{Generalized Methodology To Obtain Modified Topology}

The algorithm for the proposed methodology involves the following steps.

Step-1: In this step, the methodology initially considers the DFA predicates that are extracted from the CAD environment as input.

Step-2: In this step, by considering the DFA predicates as input, the methodology identifies the contact information between the parts. 
Step-3: In this step, the methodology checks the material properties, functionality properties and relative motion properties to the contact parts.

Step-4: In this step, merging of the parts takes place between those satisfies the properties mentioned in the step-3. After merging, a new modified topology of the assembly with reduced number of levels is obtained. The complete details of merging of the parts are given in the results section.

To validate the proposed methodology, an industrial product (Boothroyd motor assembly) with 10 parts (4 primary parts and 6 secondary parts) has been considered as shown in the Figure 1.

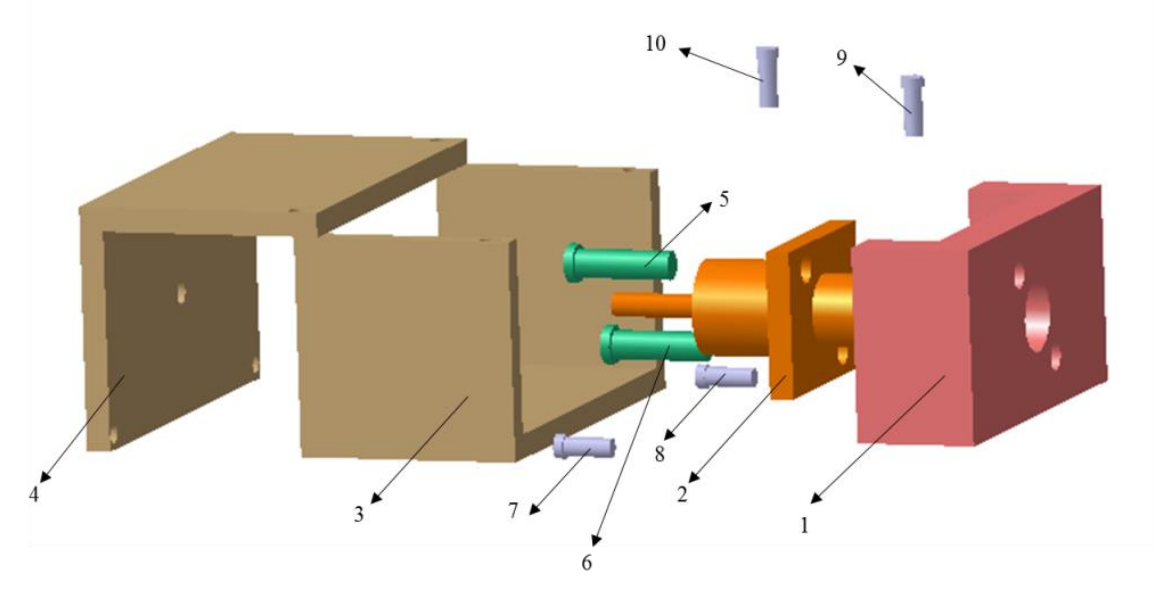

Figure 1. Boothroyd motor assembly [4]

1-Base, 2- Motor, 3-Bottom cover, 4- Front cover, $5 \&$ 6- Motor nuts, 7, 8, 9\&10-Cover nuts

The motor assembly consists of ten parts, in which four are primary parts and six are secondary parts. The DFA predicates for the considered assembly is as follows:

\subsection{Liaison data.}

In the liaison matrix, ' 0 ' represents no contact between the parts and ' 1 ' represents contact between the parts. The liaison matrix is as follows:

\subsection{Material matrix.}

$$
\text { Liaison data }=\begin{array}{c|c|c|c|c|}
\hline & 1 & 2 & 3 & 4 \\
\hline 1 & 0 & 1 & 1 & 1 \\
\hline 2 & 1 & 0 & 0 & 1 \\
\hline 3 & 1 & 0 & 0 & 1 \\
\hline 4 & 1 & 1 & 1 & 0 \\
\hline
\end{array}
$$

In the material matrix, ' 1 ' represents the same material for the compared parts and ' 0 ' represents different material for the compared parts. The material matrix is as follows:

$$
\begin{array}{|c|c|c|c|c|}
\hline & 1 & 2 & 3 & 4 \\
\hline 1 & 0 & 0 & 0 & 0 \\
\hline 2 & 0 & 0 & 0 & 0 \\
\hline 3 & 0 & 0 & 0 & 1 \\
\hline 4 & 0 & 0 & 1 & 0 \\
\hline
\end{array}
$$

\subsection{Functionality matrix.}

In the functionality matrix, ' 1 ' represents the non-presence of disturbance between the compared parts and ' 0 ' represents the presence of disturbance between the compared parts. The functionality matrix is as follows: 


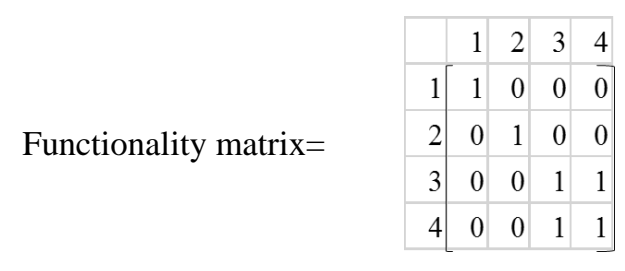

\subsection{Relative motion matrix.}

In the relative motion matrix, ' 1 ' represents the relative motion between the compared parts and ' 0 ' represents the no relative motion between the compared parts. The relative emotion matrix is as follows:

$$
\begin{array}{|c|c|c|c|c|c|}
\hline & 1 & 2 & 3 & 4 \\
\hline \multirow{3}{*}{\text { Relative motion matrix }=} & 1 & 0 & 1 & 1 & 1 \\
\hline 2 & 1 & 0 & 1 & 1 \\
\hline 3 & 1 & 1 & 0 & 0 \\
\hline 4 & 1 & 1 & 0 & 0 \\
\hline
\end{array}
$$

The detailed methodology of DFA concept is explained in Figure 2 with the help of a flow chart. DFA predicates are initially taken as input to perform parts merging operation.

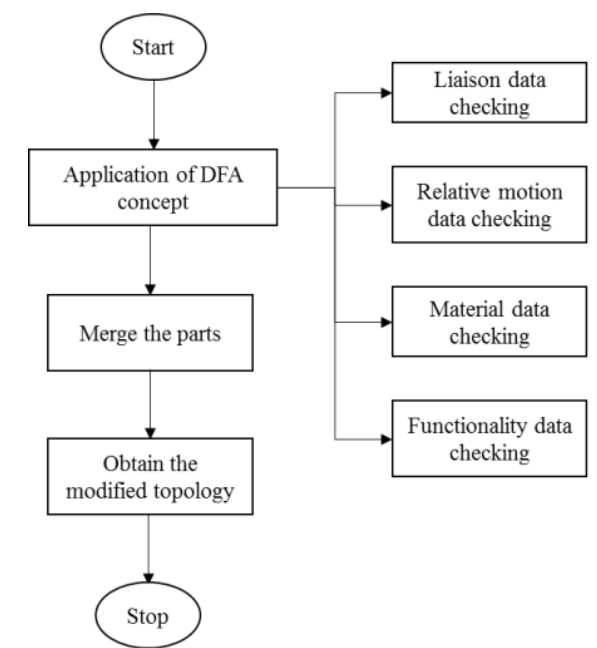

\section{Pseudo code}

Figure 2. Methodology for applying DFA concept

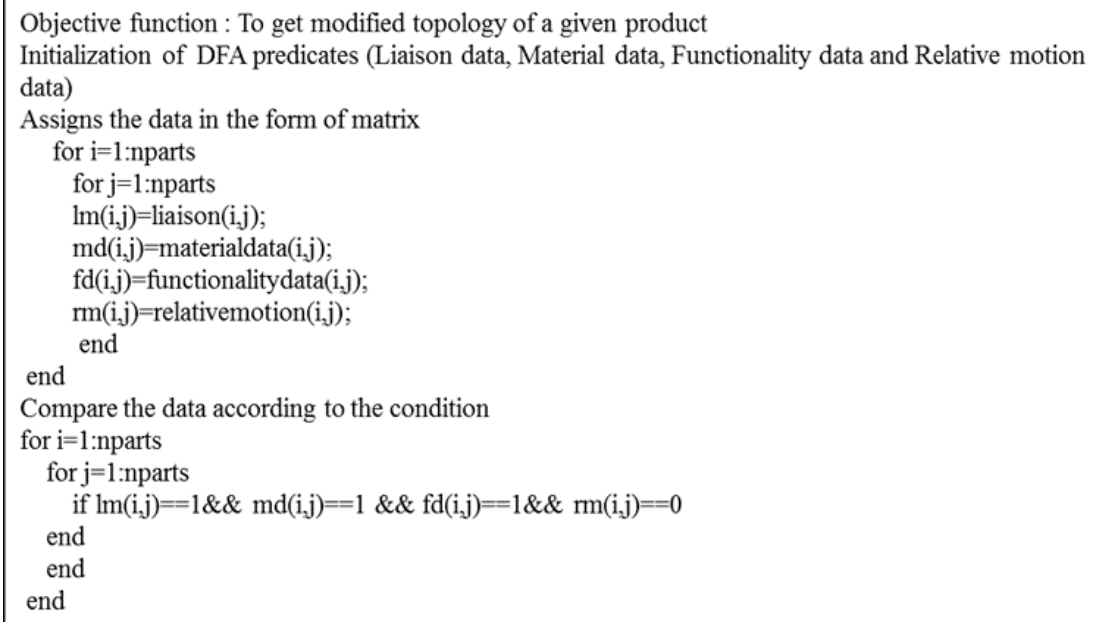

Figure 3. Pseudo code for DFA methodology 


\section{Results and Discussions}

DFA methodology considers the liaison data, material data, functionality data and relative motion data as inputs and performs merging operation. The detailed liaison graph is shown in the Figure 4.

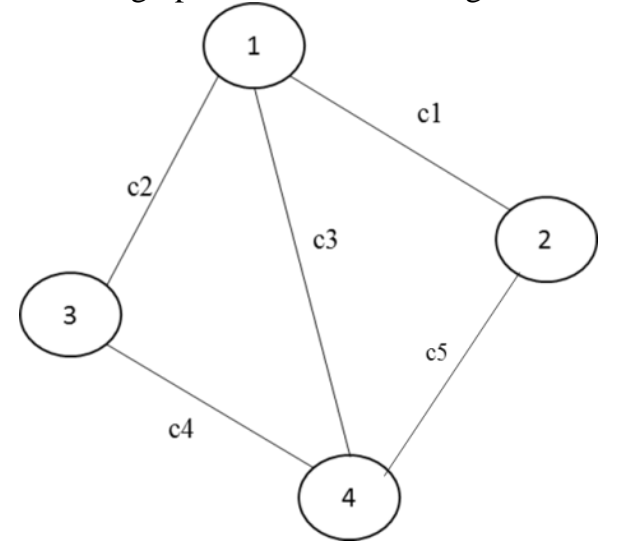

Figure 4. Liaison graph of Boothroyd motor assembly [14]

The merging of parts by DFA concept is clearly explained using liaison graph, which is shown in Table 1.

\begin{tabular}{|c|c|c|c|c|c|}
\hline Liaison graph & Connection set & $\begin{array}{c}\text { Material } \\
\text { similarity }\end{array}$ & $\begin{array}{c}\text { Functionality } \\
\text { disturbance }\end{array}$ & $\begin{array}{c}\text { Relative } \\
\text { motion } \\
\text { between the } \\
\text { parts }\end{array}$ & Remarks \\
\hline & $1-2$ & No & Yes & Yes & No merging \\
\hline & $1-3$ & No & Yes & Yes & No merging \\
\hline & $1-4$ & No & Yes & Yes & No merging \\
\hline & $2-1$ & No & Yes & Yes & No merging \\
\hline & $2-4$ & No & Yes & Yes & No merging \\
\hline & $3-1$ & No & Yes & Yes & No merging \\
\hline & $3-4$ & Yes & No & No & Merge \\
\hline & $4-1$ & No & Yes & Yes & No merging \\
\hline & $4-3$ & Yes & No & No & Merge \\
\hline
\end{tabular}

Table 1 explains the proposed DFA methodology to obtain the modified topology of a given motor assembly. The ' 3 ' level assembly has been reduced to ' 2 ' level assembly by merging the parts $3 \& 4$. The modified liaison diagram of the motor assembly is shown in the Figure 5.

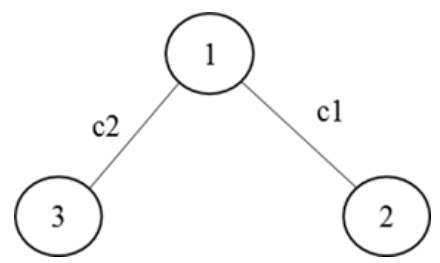

Figure 5. Modified topology of motor assembly liaison diagram 
The generated modified topology by DFA approach with reduced levels of assembly is shown in the Figure 6.
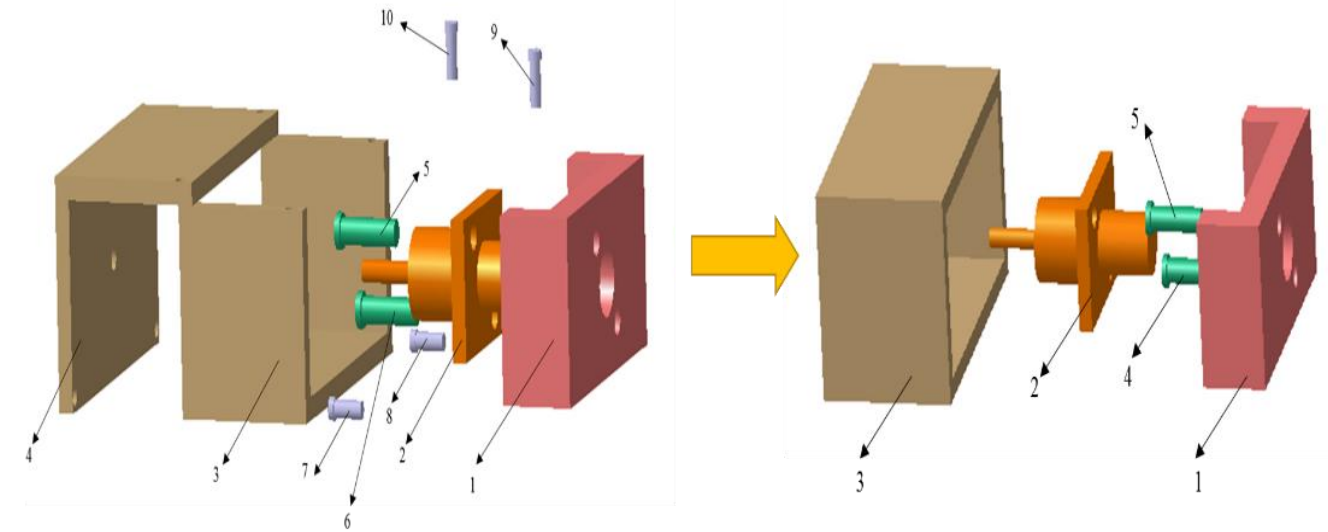

Figure 6. Modified topology of the Boothroyd motor assembly

By the application of DFA methodology, a '3' levels assembly has been reduced to '2' levels assembly. The reduction of number of levels in the assembly (10-part assembly to 5-part assembly) drastically reduces the manufacturing cost as well as the assembly efforts and time.

\section{Conclusions}

A novel strategy for DFA approach for modified topology is proposed to reduce the manufacturing cost and time for an assembled product. The proposed methodology is implemented to an industrial product 'motor drive assembly' in order to obtain the modified topology while reducing the number of assembly levels. This methodology merges the parts that satisfy the DFA predicates, by which cost of the manufacturing as well as the time and cost of the assembly drastically reduces. The proposed method successfully obtains a modified topology while satisfying all the DFA predicates. As this method is automated, there is no need of human intervention; human error is completely eradicated. This automated method reduces the number of parts of the assembly by reducing the levels of the assembly sequence. Thus lot time is saved which results the speed of manufacturing process.

\section{References}

1. M. V. A. R. Bahubalendruni, B. B. Biswal, "A review on assembly sequence generation and its automation," in Proceedings of the Institution of Mechanical Engineers, Part C: Journal of Mechanical Engineering Science, vol. 230, no. 5, pp. 824-838, 2016

2. M. V. A. R. Bahubalendruni, B. B. V. L. Deepak, and B. B. Biswal, "An advanced immune based strategy to obtain an optimal feasible assembly sequence," Assembly Automation, vol. 36, no.2, pp. 127-137, 2016.

3. B. B. Biswal, B. B. Deepak, and Y. Rao, "Optimization of robotic assembly sequences using immune based technique," Journal of Manufacturing Technology Management, vol. 24, no. 3, pp. 384-396, 2013

4. G. Boothroyd, "Design for assembly the key to design for manufacture," The International Journal of Advanced Manufacturing Technology, vol. 2, no. 3, pp. 3-11, 1987.

5. G. Boothroyd, P. Dewhurst, W. A. Knight, "Product Design for Manufacture and Assembly, revised and expanded," CRC Press, 1994.

6. R. S. Chen, K. Y. Lu, and S. C. Yu, "A hybrid genetic algorithm approach on multi-objective of assembly planning problem," Engineering Applications of Artificial Intelligence, vol. 15, no. 5, pp. 447-457, 2002.

7. T. de Fazio, and D. Whitney, "Simplified generation of all mechanical assembly sequences," IEEE Journal on Robotics and Automation vol. 3, no. 6, pp. 640-658, 1987.

8. T. Dong, "A knowledge-based approach to assembly sequence planning," The International Journal of Advanced Manufacturing Technology, vol. 32, no. 11-12, pp. 1232-1244, 2007.

9. F. Failli, G. Dini, "Ant colony systems in assembly planning: a new approach to sequence detection and optimization," in Proceedings of the 2nd CIRP International Seminar on Intelligent computation in manufacturing engineering, 2000.

10. B. Gunji, B. Deepak, M. Bahubalendruni, B. Biswal, "Hybridized genetic-immune based strategy to obtain optimal feasible assembly sequences," International Journal of Industrial Engineering Computations, vol. 8, no. 3, pp. 333-346, 2017.

11. D. S. Hong, and H. S. Cho, "Generation of robotic assembly sequences using a simulated annealing," Intelligent Robots and Systems, 1999. IROS'99. Proceedings. 1999 IEEE/RSJ International Conference on. Vol. 2. IEEE, 1999.

12. Y. Hsu, P. Tai, M. Wang, W. Chen, "A knowledge-based engineering system for assembly sequence planning," The International Journal of Advanced Manufacturing Technology, vol. 55, no. 5-8, pp. 763-782, 2011.

13. G. B. Murali, B. B. V. L. Deepak, M. V. A. R. Bahubalendruni, B. B. Biswal, "Optimal Assembly Sequence Planning Towards Design for Assembly Using Simulated Annealing Technique," 2017. 
14. H. Shan, S. Zhou and Z. Sun, "Research on assembly sequence planning based on genetic simulated annealing algorithm and ant colony optimization algorithm," Assembly Automation, vol. 29, no. 3, pp. 249-256, 2009.

15. G. C. Smith, and S. S. F. Smith. "An enhanced genetic algorithm for automated assembly planning," Robotics and ComputerIntegrated Manufacturing, vol. 18, no. 5, pp. 355-364, 2002.

16. H. E. Tseng, M. H. Chen, C. C. Chang, and W. P. Wang, "Hybrid evolutionary multi-objective algorithms for integrating assembly sequence planning and assembly line balancing," International Journal of Production Research, vol. 46, no. 21, pp. 5951-5977, 2008.

17. H. Wong, M. C. Leu, "Adaptive genetic algorithm for optimal printed circuit board assembly planning," CIRP AnnalsManufacturing Technology, vol. 42, no. 1, pp. 17-20, 1993.

18. X. F. Zha, S. Y. E. Lim, and S. C. Fok. "Integrated knowledge-based assembly sequence planning," The International Journal of Advanced Manufacturing Technology, vol. 14, no. 1, pp: 50-64, 1998. 